\title{
Sparse Planar Array Synthesis Using Matrix Enhancement and Matrix Pencil
}

\author{
Mei-yan Zheng, ${ }^{1}$ Ke-song Chen, ${ }^{1}$ Hong-gang Wu, ${ }^{2}$ and Xian-pan Liu ${ }^{3}$ \\ ${ }^{1}$ School of Electronic Engineering, University of Electronic Science and Technology of China, Chengdu 611731, China \\ ${ }^{2}$ The Second Research Institute of Civil Aviation of China, Chengdu 610041, China \\ ${ }^{3}$ School of Astronautics and Aeronautics, University of Electronic Science and Technology of China, Chengdu 611731, China
}

Correspondence should be addressed to Mei-yan Zheng; zhengmeiyan123456@126.com

Received 3 July 2012; Accepted 28 January 2013

Academic Editor: Tat Yeo

Copyright (C) 2013 Mei-yan Zheng et al. This is an open access article distributed under the Creative Commons Attribution License, which permits unrestricted use, distribution, and reproduction in any medium, provided the original work is properly cited.

\begin{abstract}
The matrix enhancement and matrix pencil (MEMP) plays important roles in modern signal processing applications. In this paper, MEMP is applied to attack the problem of two-dimensional sparse array synthesis. Firstly, the desired array radiation pattern, as the original pattern for approximating, is sampled to form an enhanced matrix. After performing the singular value decomposition (SVD) and discarding the insignificant singular values according to the prior approximate error, the minimum number of elements can be obtained. Secondly, in order to obtain the eigenvalues, the generalized eigen-decomposition is employed on the approximate matrix, which is the optimal low-rank approximation of the enhanced matrix corresponding to sparse planar array, and then the ESPRIT algorithm is utilized to pair the eigenvalues related to each dimension of the planar array. Finally, element positions and excitations of the sparse planar array are calculated according to the correct pairing of eigenvalues. Simulation results are presented to illustrate the effectiveness of the proposed approach.
\end{abstract}

\section{Introduction}

The question of nonuniform planar array synthesis with fewer number of elements for a desired beam pattern has been a popular concern since early in the 1970s. Reducing the number of elements in planar arrays has long been a problem both practical and theoretical significance being the subject of concern; for example, satellite communication system has limitation on the weight of antenna systems and subsystem accessories which wishes to use the minimum number of elements. Furthermore, fewer elements can lower the cost obviously and simplify the antenna system.

The synthesis of nonuniform array elements' positions, excitations, and phases is a complicated nonlinear problem which contains a number of decision variables. So far, there have been many technologies used to synthesize the nonuniform arrays, including optimization algorithm (such as genetic algorithm (GA) [1], differential evolution algorithm (DEA) [2], analytical methods [3, 4], matrix pencil method (MPM) [5], dynamic programming, and particle swarm optimization (PSO)) and other comprehensive technologies.
However, some optimization algorithms such as GA, DEA, and PSO are suitable for solving nonlinear optimization of high dimension complex function, but they are very time consuming for their intrinsic iterative mechanism. Many other comprehensive approaches based on iteration do not guarantee that all variables are global optimal solutions. The new non iterative methods, called MPM [6,7] and MEMP [8], have been successfully used by Yingbo Hua, Tapan K. S. and Odilon Pererira in the parameters estimation of a sum of complex exponentials, such as two-dimensional frequencies [8], azimuth/elevation angel [9], and two-dimensional DOA [10]. These years Liu and others find a new application in the synthesis of nonuniform array for MPM $[5,11]$.

MPM is qualified to the synthesis of sparse planar array which is designed to a separable planar array by a product of two orthogonal linear sparse arrays [5]. It can guarantee the best features in the two principal plane patterns in this particular case, but it cannot at the same time guarantee that others have the same characteristics.

Further expansion of MPM to the synthesis of inseparable distribution sparse planar array in this paper, just as in signal 
processing applications, the new method similarly called matrix enhancement and matrix pencil (MEMP) in synthesis of sparse arrays. The organization of this paper is listed as follows. In Section 2, the optimal array model is presented. In Section 3, the idea of matrix enhancement and matrix pencil is introduced, and then the method will be applied to estimate the minimum number of elements and to calculate the locations and excitations of the new array. Section 4 introduces the essential steps of MEMP. Some numerical results are presented in Section 5 to show the efficiency of the proposed method. Finally, some conclusions are drawn in Section 6.

\section{The Optimal Array Model}

Assume an array of $N$ discrete similarly oriented identical elements arranged arbitrarily in a 3-D space, as shown in Figure 1. Denote the excitation in the $i$ th element located at $\left(r_{i}, \theta_{i}, \phi_{i}\right)$ as $R_{i}$.

According to the superposition theorem of electromagnetic waves, we can write the following array factor [12]:

$$
F(\theta, \varphi)=\sum_{i=1}^{N} R_{i} \exp \left[j\left(\phi_{i}+k r_{i} \cos \alpha_{i}\right)\right],
$$

where $k=2 \pi / \lambda, \lambda$ is the wavelength. Consider,

$$
\cos \alpha_{i}=\sin \theta \sin \theta_{i} \cos \left(\phi-\phi_{i}\right)+\cos \theta \cos \theta_{i}
$$

where $0 \leq \theta \leq \pi$, and $0 \leq \phi \leq 2 \pi$ are the azimuth and elevation, respectively.

For the planar array, (1) can be simplified as

$$
F(u, v)=\sum_{i=1}^{N} R_{i} \exp \left[j k\left(d_{x} u+d_{y} v\right)\right],
$$

where $d_{x}$ and $d_{y}$ are the abscissa and ordinate of the $i$ th element, and $u=\sin \theta \cos \phi, v=\sin \theta \sin \phi$.

\section{MEMP to Synthesize Planar Array}

3.1. Estimation of the Minimum Number of Elements. Matrix enhancement and matrix pencil is to use as few elements as possible to form a new planar array to approximate the desired pattern. Thus, the optimal mathematical is described as

$$
\begin{aligned}
& \min \{Q\} \\
& \text { st. }\left\{\operatorname{Min}\left\|F(u, v)-\sum_{i=1}^{Q} R_{i}^{\prime} \exp \left[j k\left(d_{x}^{\prime} u+d_{y}^{\prime} v\right)\right]\right\|\right\} \leq \varepsilon,
\end{aligned}
$$

where $R_{i}^{\prime}, d_{x}^{\prime}$, and $d_{y}^{\prime}$ are the excitation, abscissa, and ordinate of the new planar array. $L=2$ because this paper uses the total least squares criteria.

Firstly, to sample the desired pattern from $u=-1, v=-1$ to $u=1, v=1$ uniformly, then $u_{m}=m \Delta=m / N, m=$ $-N, \ldots, 0, \ldots, N ; v_{n}=n \Delta=n / N, n=-N, \ldots, 0, \ldots, N$, which has $(2 N+1)(2 N+1)$ sampling points.

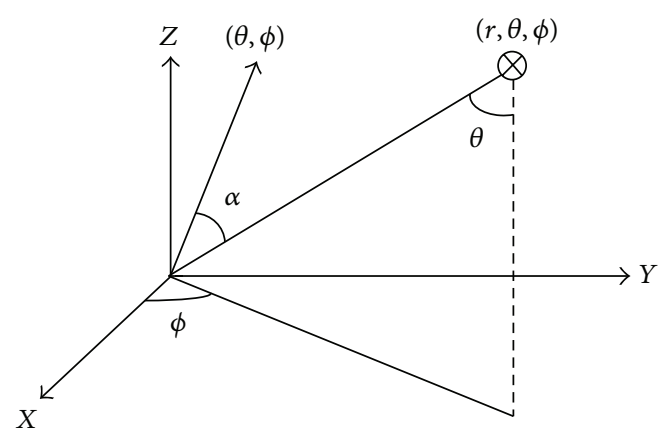

FIGURE 1: Reference coordinates of an arbitrary array.

The value of any sampling point is

$$
f(m, n)=\sum_{i=1}^{N} R_{i} y_{i}^{m} z_{i}^{n}
$$

where $y_{i}=e^{j k d_{x} \Delta}$ and $z_{i}=e^{j k d_{y} \Delta}$.

Secondly, to construct an enhanced matrix $\mathbf{X}_{e}$ using the sampling point data,

$$
\mathbf{X}_{e}=\left[\begin{array}{cccc}
\mathbf{X}_{0} & \mathbf{X}_{1} & \cdots & \mathbf{X}_{2 N-K+1} \\
\mathbf{X}_{1} & \mathbf{X}_{2} & \cdots & \mathbf{X}_{2 N-K+2} \\
\vdots & \vdots & & \vdots \\
\mathbf{X}_{K-1} & \mathbf{X}_{K} & \cdots & \mathbf{X}_{2 N}
\end{array}\right]
$$

where

$$
\mathbf{X}_{m}=\left[\begin{array}{cccc}
x(m ; 0) & x(m ; 1) & \cdots & x(m ; 2 N-L+1) \\
x(m ; 1) & x(m ; 2) & \cdots & x(m ; 2 N-L+2) \\
\vdots & \vdots & & \vdots \\
x(m ; L-1) & x(m ; L) & \cdots & x(m ; 2 N)
\end{array}\right],
$$

where $x(m ; n)=f(m-N ; n-N)$. $\mathbf{X}_{e}$ is a Hankel block matrix and $\mathbf{X}_{m}$ is a Hankel matrix. The choice of parameters $\{K, L\}$ must satisfy the following conditions:

$$
\begin{aligned}
& (K-1) L \geq N, \\
& K(L-1) \geq N,
\end{aligned}
$$

$$
(2 N+2-K)(2 N+2-L) \geq N \text {. }
$$

Thirdly, to perform the singular value decomposition (SVD) of the enhanced matrix $\mathbf{X}_{e}$,

$$
\begin{aligned}
\mathbf{X}_{e} & =\sum_{i=1}^{\min } \sigma_{i} \mathbf{u}_{i} v_{i}^{H} \\
& =\mathbf{U}_{s} \Sigma_{s} \mathbf{V}_{s}^{H}+\mathbf{U}_{n} \Sigma_{n} \mathbf{V}_{n}^{H}
\end{aligned}
$$

$\min =\min \{K L,(2 N+2-K)(2 N+2-L)\}$ which is the smaller dimension of $\mathbf{X}_{e}$, and $\mathbf{U}_{s}, \Sigma_{s}$, and $\mathbf{V}_{S}$ contain the $N$ principal 
eigenvalues and eigenvectors. $\mathbf{U}_{n}, \Sigma_{n}$, and $\mathbf{V}_{n}$ contain the remaining eigenvalues and eigenvectors. Specifically,

$$
\begin{gathered}
\mathbf{U}_{s}=\left[\mathbf{u}_{1}, \mathbf{u}_{2}, \ldots, \mathbf{u}_{N}\right], \\
\Sigma_{s}=\operatorname{diag}\left\{\sigma_{1}, \sigma_{1}, \ldots, \sigma_{N}\right\}, \\
\mathbf{V}_{S}=\left[v_{1}, v_{2}, \ldots, v_{N}\right], \\
\mathbf{U}_{n}=\left[\mathbf{u}_{N+1}, \mathbf{u}_{N+2}, \ldots, \mathbf{u}_{\min }\right], \\
\Sigma_{n}=\operatorname{diag}\left\{\sigma_{N+1}, \sigma_{N+2}, \ldots, \sigma_{\min }\right\}, \\
\mathbf{V}_{n}=\left[v_{N+1}, v_{N+2}, \ldots, v_{\min }\right],
\end{gathered}
$$

where $\sigma_{1} \geq \sigma_{2} \geq \cdots \geq \sigma_{\min }$. The rank of enhanced matrix $\mathbf{X}_{e}$ is equal to the number of nonzero singular values. Generally, an array of $N$ elements has $N$ non-zero singular values; that is, $\sigma_{i}>0(i=1, \ldots, N), \sigma_{i}=0(i>N+1)$. So, $\Sigma_{n}$ is equal to zero. Equation (9) can be simplified to

$$
\mathbf{X}_{e}=\mathbf{U}_{s} \Sigma_{s} \mathbf{V}_{s}^{H} \text {. }
$$

However, some literature results [13] show that the number of important singular values is much smaller than the total number of array elements, that is the unimportant elements' contributions can be replaced by a combination of other elements. Therefore, we can discard the unimportant singular values to get a low-rank approximation of the enhanced matrix, which corresponds to the new array with fewer elements. The usual approach is to set these small singular values to zero. That is,

$$
\mathbf{X}_{Q}=\mathbf{U}_{s} \Sigma_{Q} \mathbf{V}_{s}^{H},
$$

where $\Sigma_{Q}=\operatorname{diag}\left\{\sigma_{1}, \sigma_{1}, \ldots, \sigma_{Q}, 0, \ldots, 0\right\}, Q \leq N$.

In the actual synthesis of array, the minimum value of $Q$ can be determined by the following formula [11]:

$$
Q=\min \left\{q ; \mid \frac{\sqrt{\sum_{i=q+1}^{N} \sigma_{i}^{2}}}{\sqrt{\sum_{i=1}^{q} \sigma_{i}^{2}}}<\varepsilon\right\},
$$

where $\varepsilon$ is a small positive number, and the choice of $\varepsilon$ depends on the approximation between the reconstructed pattern and the original pattern.

3.2. The Solutions of Eigenvalues $y_{i}$ and $z_{i}$. The eigenvalues are obtained by constructing two matrixs and solving the generalized eigenvalue of the matrixes $[7,8]$, then utilizing the ESPRIT algorithm in the literature [14] and pairing algorithm to estimate the correct pairing of $y_{i}$ and $z_{i}$.

3.2.1. Extracting $y_{i}$. Once the low rank matrix $\mathbf{X}_{\mathrm{Q}}$ is obtained, to solve the eigenvalue $y_{i}$ is equal to solve the generalized eigenvalue of the following equation:

$$
\left(\mathbf{X}_{Q, f}-y \mathbf{X}_{Q, l}\right) v=0
$$

where $\mathbf{X}_{\mathrm{Q}, f}$ and $\mathbf{X}_{\mathrm{Q}, l}$ are obtained by deleting the first $L$ rows and the last $L$ rows of $\mathbf{X}_{Q}$. To solve (14) is equal to solve the generalized eigenvalue of the following equation:

$$
\left(\mathbf{U}_{2}-y \mathbf{U}_{1}\right) v=0 \text {, }
$$

where $\mathbf{U}_{2}$ and $\mathbf{U}_{1}$ are obtained by deleting the first $L$ rows and the last $L$ rows of $U_{Q}$ which contains only $Q$ dominant leftsingular vectors of $\mathbf{U}_{s}$ in (11).

Therefore, it can be obviously observed that the matrix pencil $\mathbf{U}_{2}-y \mathbf{U}_{1}$ can be expressed as

$$
\left(\mathbf{U}_{2}-y \mathbf{U}_{1}\right)=\mathbf{E}_{1}\left(\mathbf{Y}_{d}-y \mathbf{I}\right) \mathbf{T},
$$

where $\mathbf{Y}_{d}$ is a diagonal matrix composed of the eigenvalues of $\left\{y_{i}, i=1, \ldots, Q\right\}$.

3.2.2. Extracting $z_{i}$. In order to extract another set of $\left\{z_{i}, i=\right.$ $1, \ldots, Q\}$, firstly introduce a permutation matrix $\mathbf{P}$ as

$$
\begin{aligned}
\mathbf{P}=[ & \mathbf{p}^{T}(1), \mathbf{p}^{T}(1+L), \ldots, \mathbf{p}^{T}(1+(K-1) L), \\
& \mathbf{p}^{T}(2), \mathbf{p}^{T}(2+L), \ldots, \\
& \mathbf{p}^{T}(1+(K-1) L), \ldots, \ldots, \mathbf{p}^{T}(L), \\
& \left.\mathbf{p}^{T}(L+L), \ldots, \mathbf{p}^{T}(1+(K-1) L)\right]^{T} .
\end{aligned}
$$

The element $\mathbf{p}(i)$ is a $K L \times 1$ vector with one at the $i$ th position and zero everywhere else. Left multiplying $\mathbf{U}_{s}$ by $\mathbf{P}$, we can get

$$
\mathbf{U}_{s P}=\mathbf{P} \mathbf{U}_{s} .
$$

According to (15), we can conclude that to solve the eigenvalues $z_{i}$ is equal to solve the generalized eigenvalues of the following equation:

$$
\left(\mathbf{U}_{2 P}-z \mathbf{U}_{1 P}\right) v=0,
$$

where $\mathbf{U}_{2 P}$ and $\mathbf{U}_{1 P}$ are obtained by deleting the first $K$ rows and the last $K$ rows of $\mathbf{U}_{\mathrm{QP}}$ which contains only $Q$ dominant left-singular vectors of $\mathbf{U}_{s P}$ in (18).

Therefore, it can be obviously observed that the matrix pencil $\mathbf{U}_{2 P}-z \mathbf{U}_{1 P}$ can be expressed as

$$
\left(\mathbf{U}_{2 P}-z \mathbf{U}_{1 P}\right)=\mathbf{E}_{1 P}\left(\mathbf{Z}_{d}-z \mathbf{I}\right) \mathbf{T},
$$

where $\mathbf{Z}_{d}$ is a diagonal matrix composed of the eigenvalues of $\left\{z_{i}, i=1, \ldots, Q\right\}$.

3.2.3. Pairing. According to (16) and (20), we can deduce that

$$
\begin{gathered}
\mathbf{F}_{1}=\mathbf{U}_{1}^{+} \mathbf{U}_{2}=\mathbf{T}^{-1} \mathbf{Y}_{d} \mathbf{T} \\
\mathbf{F}_{2}=\mathbf{U}_{1 P}^{+} \mathbf{U}_{2 P}=\mathbf{T}^{-1} \mathbf{Z}_{d} \mathbf{T} .
\end{gathered}
$$

It can be seen from (21) that the pairing information of the eigenvalues is included in $\mathbf{T}$. 


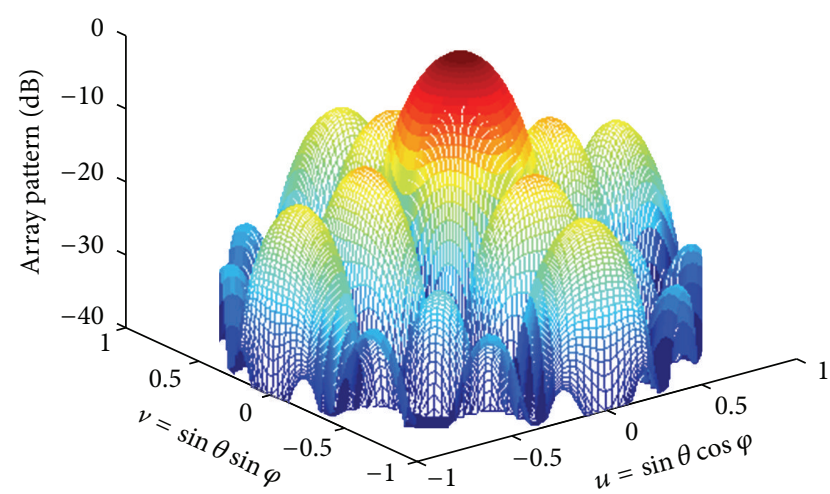

(a)

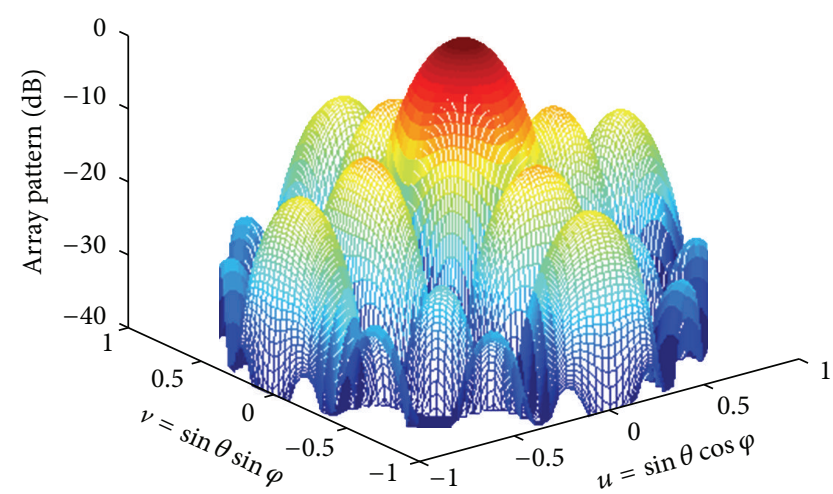

(b)

Figure 2: (a) The desired radiation pattern. (b) The reconstructed radiation pattern.

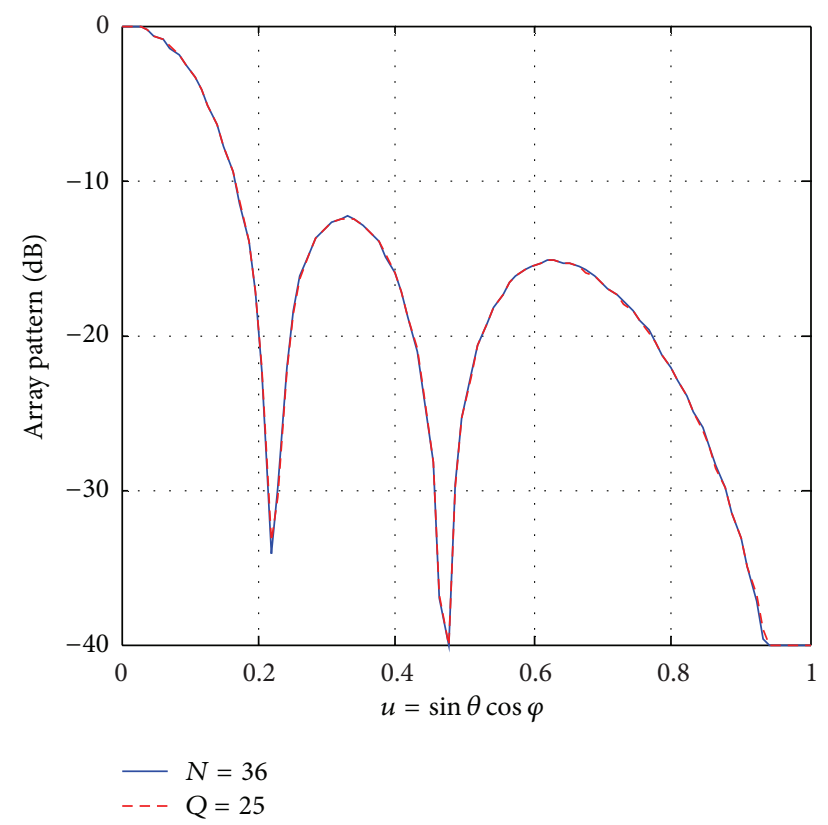

FIGURE 3: The cross-section of the pattern.

The eigenvalue decomposition of a linear combination of $\mathbf{F}_{1}$ and $\mathbf{F}_{2}$ is computed to obtain a transformation diagonalizing of these two matrixs as follows:

$$
\beta \mathbf{F}_{1}+(1-\beta) \mathbf{F}_{1}=\mathbf{T}^{-1} \mathbf{D T} \text {. }
$$

The transformation $\mathbf{T}$ is applied to $\mathbf{F}_{1}$ and $\mathbf{F}_{2}$ to obtain the matrixs:

$$
\mathbf{Y}_{d}=\mathbf{T F}_{1} \mathbf{T}^{-1}, \quad \mathbf{Z}_{d}=\mathbf{T F}_{2} \mathbf{T}^{-1}
$$

The values in the diagonal position of $\mathbf{Y}_{d}$ and $\mathbf{Z}_{d}$ determine the pairing.

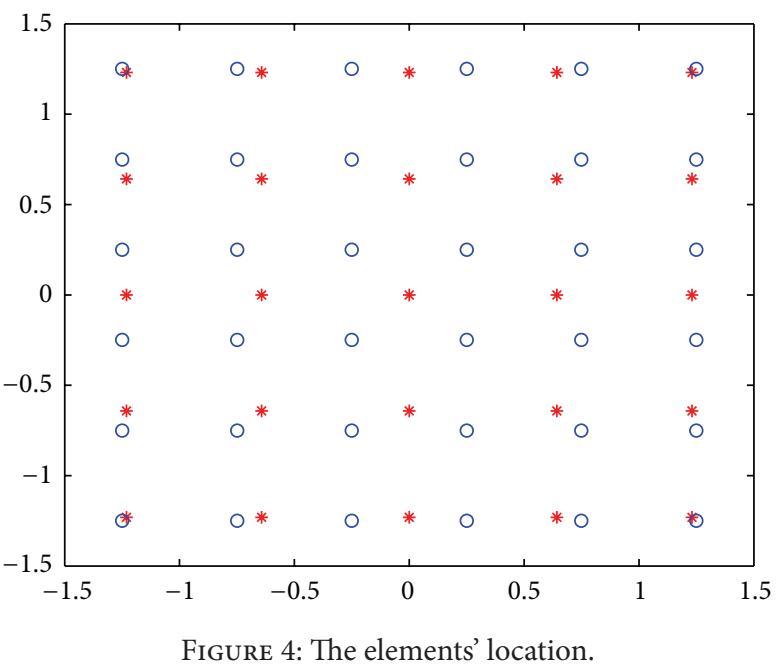

3.3. To Solve Elements' Excitations and Locations. Once $y_{i}$ and $z_{i}$ are obtained, the locations can be solved according to (14) in the literature [13]; that is,

$$
\begin{aligned}
& d_{x}^{\prime}=\frac{1}{j k \Delta} \ln \left(y_{i}\right), \\
& d_{y}^{\prime}=\frac{1}{j k \Delta} \ln \left(z_{i}\right) .
\end{aligned}
$$

As pointed in [9], a variety of synthetical examples show that, for the symmetrical pencil beam pattern, the imaginary parts of $d_{i}^{\prime}$ are much smaller than the real parts. For example, the imaginary parts of $d_{i}^{\prime}$ are ten orders smaller than the real parts for the synthesis of traditional Chebyshev planar pattern. Under this case, we can use the real parts of $d_{i}^{\prime}$ directly. The elements' locations are calculated as follows:

$$
\begin{aligned}
& d_{x}^{\prime}=\frac{1}{j k \Delta} \ln \left(\widehat{y}_{i}\right), \\
& d_{y}^{\prime}=\frac{1}{j k \Delta} \ln \left(\widehat{z}_{i}\right),
\end{aligned}
$$




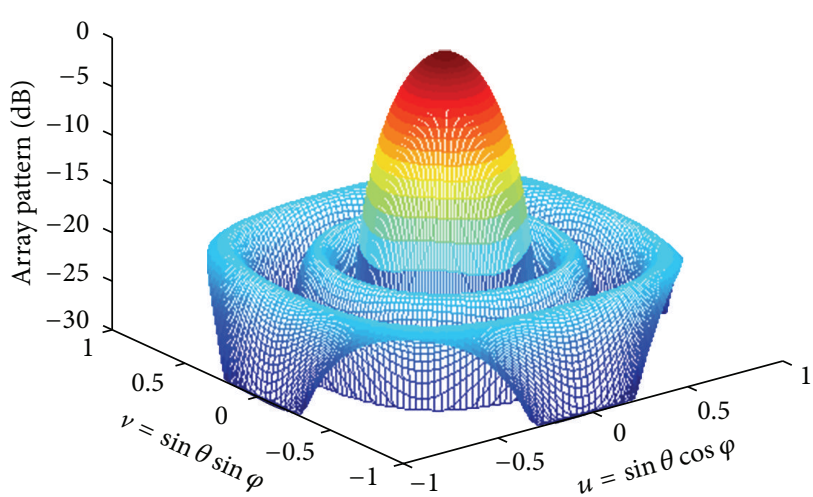

(a)

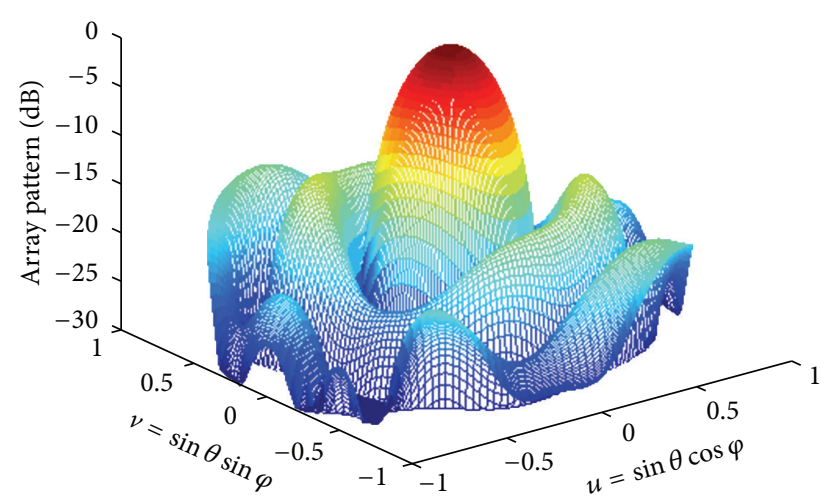

(b)

FIgURE 5: (a) The Chebyshev radiation pattern. (b) The reconstructed radiation pattern.

where

$$
\widehat{y}_{i}=\frac{y_{i}}{\left|y_{i}\right|}, \quad \widehat{z}_{i}=\frac{z_{i}}{\left|z_{i}\right|}
$$

The elements' excitations can be obtained as follows:

$$
\mathbf{R}=\mathbf{E}_{L}^{+} \mathbf{X}_{e} \mathbf{E}_{R}^{+} \text {. }
$$

The diagonal elements of matrix $\mathbf{R}$ are $R_{i}(i=1, \ldots, Q)$ where

$$
\begin{aligned}
& \mathbf{E}_{L}^{+}=\left(\mathbf{E}_{L}^{H} \mathbf{E}_{L}\right)^{-1} \mathbf{E}_{L}^{H}, \\
& \mathbf{E}_{R}^{+}=\mathbf{E}_{R}^{H}\left(\mathbf{E}_{R} \mathbf{E}_{R}^{H}\right)^{-1},
\end{aligned}
$$

where

$$
\begin{gathered}
\mathbf{E}_{L}=\left[\begin{array}{c}
\mathbf{Z}_{L} \mathbf{Y}_{d}^{-N} \\
\mathbf{Z}_{L} \mathbf{Y}_{d}^{-N+1} \\
\vdots \\
\mathbf{Z}_{L} \mathbf{Y}_{d}^{K-N-1}
\end{array}\right], \\
\mathbf{E}_{R}=\left[\mathbf{Z}_{R}, \mathbf{Y}_{d} \mathbf{Z}_{R}, \ldots, \mathbf{Y}_{d}^{2 N-K+1} \mathbf{Z}_{R}\right],
\end{gathered}
$$

where

$$
\begin{gathered}
\mathbf{Z}_{L}=\left[\begin{array}{cccc}
\left(\widehat{z}_{1}\right)^{-N} & \left(\widehat{z}_{2}\right)^{-N} & \cdots & \left(\widehat{z}_{\mathrm{Q}}\right)^{-N} \\
\left(\widehat{z}_{1}\right)^{-N+1} & \left(\widehat{z}_{2}\right)^{-N+1} & \cdots & \left(\widehat{z}_{\mathrm{Q}}\right)^{-N+1} \\
\vdots & \vdots & & \vdots \\
\left(\widehat{z}_{1}\right)^{L-1-N} & \left(\widehat{z}_{2}\right)^{L-1-N} & \cdots & \left(\widehat{z}_{\mathrm{Q}}\right)^{L-1-N}
\end{array}\right], \\
\mathbf{y}_{d}=\operatorname{diag}\left\{\widehat{y}_{1}, \widehat{y}_{2}, \ldots, \widehat{y}_{\mathrm{Q}}\right\} \\
\mathbf{Z}_{R}=\left[\begin{array}{cccc}
1 & \widehat{z}_{1} & \cdots & \left(\widehat{z}_{1}\right)^{2 N-L+1} \\
1 & \widehat{z}_{2} & \cdots & \left(\widehat{z}_{2}\right)^{2 N-L+1} \\
\vdots & \vdots & & \vdots \\
1 & \widehat{z}_{\mathrm{Q}} & \cdots & \left(\widehat{z}_{\mathrm{Q}}\right)^{2 N-L+1}
\end{array}\right] .
\end{gathered}
$$

\section{The Steps of MEMP}

The essential steps of MEMP are summarized as follows.

Step 1. To sample the desired pattern uniformly and to construct an enhanced matrix $\mathbf{X}_{e}$ by the sampling data; $\mathbf{X}_{e}$ is shown as (6).

Step 2. To perform the singular value decomposition (SVD) of enhanced matrix $\mathbf{X}_{e}$ and to calculate the singular values and left singular vector $\mathbf{U}_{s}$.

Step 3. According to (13), to determine the minimum number of elements $Q$.

Step 4. According to (16) and (20), to extract the eigenvalues $y_{i}$ and $z_{i}$, then to pair them as (22).

Step 5. According to (25) and (27), to solve the elements' excitations and locations.

\section{Simulation Results}

In order to illustrate the effectiveness of MEMP, this paper gives two examples to reduce the number of elements and let the reconstructed array keep the characteristics of the original array.

5.1. Synthesis of Planar Array with Excitation of One. Suppose, there is a rectangular planar array with $6 \times 6$ elements. The elements are arranged in a rectangular grid, $d_{x}=d_{y}=\lambda / 2$, and the elements' excitations are one. In this example, there are $(2 \times 36+1)(2 \times 36+1)$ sampling points (by further increasing the sampling points will not improve the approximate accuracy of the pattern). The parameters $K=L=37$. According to (13), the required minimum number of elements is $Q=25$ when $\varepsilon=10^{-5}$. The desired pattern and the reconstructed pattern are shown in Figure 2. The obtained sidelobe levels are $-13.1525 \mathrm{~dB}$ and $-13.1522 \mathrm{~dB}$, respectively. Figure 3 compares the cross-section of the reconstructed pattern and the desired pattern. The figure shows that the array with $Q=25$ unequally spaced distribution of the elements can basically 
TABLE 1: The locations (excitations) of uniformly and nonuniformly spaced arrays.

\begin{tabular}{lccccc}
\hline \multicolumn{2}{c}{ The locations of uniformly spaced array } & \multicolumn{3}{c}{ The locations (excitations) of nonuniformly spaced array } \\
\hline $0.25,1.25$ & $0.75,1.25$ & $1.25,1.25$ & $0,1.2308(1.4579)$ & $0.6423,1.2308(1.3936)$ & $1.2308,1.2308(1.2226)$ \\
$0.25,0.75$ & $0.75,0.75$ & $1.25,0.75$ & $0,0.6423(1.6619)$ & $0.6423,0.6423(1.5886)$ & $1.2308,0.6423(1.3936)$ \\
$0.25,0.25$ & $0.75,0.25$ & $1.25,0.25$ & $0,0(1.7386)$ & $0.6423,0(1.6619)$ & $1.2308,0(1.4579)$ \\
\hline
\end{tabular}

TABLE 2: The locations (excitations) of Chebyshev and nonuniformly spaced array.

\begin{tabular}{|c|c|c|c|c|}
\hline \multicolumn{5}{|c|}{ The locations (excitations) of Chebyshev planar array } \\
\hline $0.25,1.25(0.3654)$ & & $0.75,1.25(0.1822)$ & & $1.25,1.25(0.0364)$ \\
\hline $0.25,0.75(0.2640)$ & & $0.75,0.75(0.3917)$ & & $1.25,0.75(0.1822)$ \\
\hline $0.25,0.25(0.4503)$ & & $0.75,0.25(0.2640)$ & & $1.25,0.25(0.3645)$ \\
\hline \multicolumn{5}{|c|}{ The locations (excitations) of nonuniform planar array } \\
\hline$-1.2319,-1.2463(0.0538)$ & $-0.6505,-1.2237(0.3215)$ & $-0.0914,-1.1991(0.7001)$ & $0.3336,-1.1513(0.8382)$ & $0.9598,-0.9605(0.5520)$ \\
\hline$-1.2237,-0.7295(0.2870)$ & $-0.6472,-0.6505(0.6144)$ & $0,-0.6238(0.6207)$ & $0.6472,-0.5680(0.9560)$ & $1.2237,-0.3341(0.5179)$ \\
\hline$-1.1513,-0.2376(0.6953)$ & $-0.5680,0(0.9462)$ & $0,0(0.7766)$ & $0.5680,0(0.8475)$ & $1.1513,0.2376(0.3763)$ \\
\hline$-1.1981,0.3341(0.6218)$ & $-0.5971,0.6505(0.6486)$ & $0,0.5680(0.8748)$ & $0.5971,0.7295(0.8196)$ & $1.1981,0.6238(0.6182)$ \\
\hline$-0.9598,0.9605(0.5523)$ & $-0.3336,1.2237(0.6272)$ & $0.0914,1.1991(0.7050)$ & $0.6505,1.1513(0.4298)$ & $1.2319,1.2463(0.0526)$ \\
\hline
\end{tabular}

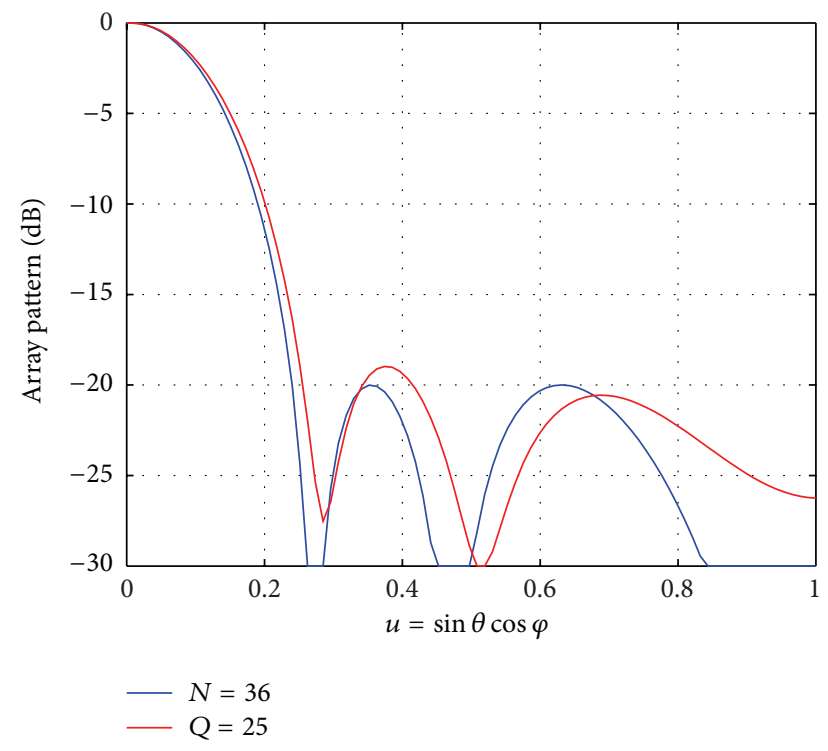

FIGURE 6: The cross section of the pattern.

reconstruct the equally spaced distribution of the elements $N=36$. The example is to save $30 \%$ of the array elements. The elements' locations of the equally and unequally spaced are shown in Figure 4. The equally spaced elements' locations and the unequally spaced elements' excitations and locations are shown in Table 1. Because the locations and excitations are symmetrically distributed, only the locations and excitations in the first quadrant are given.

5.2. Synthesis of Chebyshev Planar. Suppose that there is a chebyshev planar array with $6 \times 6$ elements. The elements are arranged in a rectangular grid, $d_{x}=d_{y}=\lambda / 2$, and the required sidelobe level is $-20 \mathrm{~dB}$. In this example, there are $(2 \times 36+1)(2 \times 36+1)$ sampling points, and the parameters

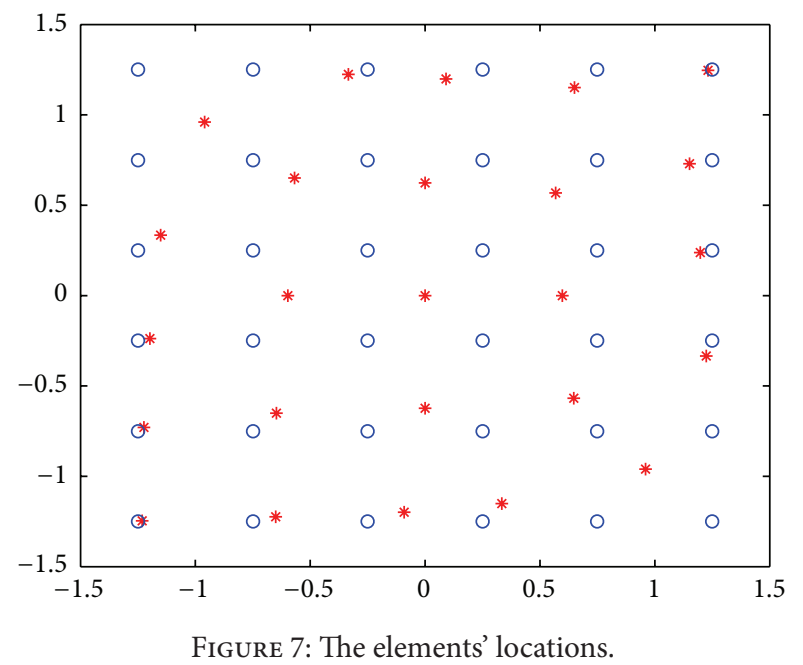

$K=L=37$. Chebyshev planar array has the limitation of the number of elements in every row which is equal to the number of elements in every column which so, we use $5 \times 5$ elements to approximate the desired Chebyshev planar array. The desired pattern and the reconstructed pattern are shown in Figure 5. The obtained sidelobe level is $-19.3058 \mathrm{~dB}$. Figure 6 compares the cross section of the reconstructed pattern with the desired pattern. Although there is a slight elevation $(0.694 \mathrm{~dB})$ of the sidelobe level, we can save $30 \%$ elements. The elements' locations of the equally and unequally spaced are shown in Figure 7. The elements' excitations and locations of equally and unequally spaced arrays are shown in Table 2.

\section{Conclusion}

A synthesis method to sparse planar array which is based on MEMP is presented. Compared to stochastic optimization 
algorithm, MEMP is a non-iterative algorithm, which is suitable for designing of sparse array with the requirements of narrow beam, low sidelobe level. In addition, compared to MPM, MEMP can guarantee the best features of either plane. The synthesis of planar array in the paper does not consider the mutual coupling $[15,16]$ between elements. But there exists mutual coupling between actual elements. Therefore, the synthesis results gotten under the condition of unconsidering mutual coupling can be solved by adjusting the elements' excitations or locations. The discussion of this paper enriches the application of MEMP to the synthesis of planar array and provides a valuable reference in engineering applications dealing with complexity of problems.

\section{Acknowledgment}

This work was supported by the National Natural Science Foundation of China (nos. 60736045 and 60702070).

\section{References}

[1] K. Chen, X. Yun, Z. He, and C. Han, "Synthesis of sparse planar arrays using modified real genetic algorithm," IEEE Transactions on Antennas and Propagation, vol. 55, no. 4, pp. 1067-1073, 2007.

[2] D. G. Kurup, M. Himdi, and A. Rydberg, "Synthesis of uniform amplitude unequally spaced antenna arrays using the differential evolution algorithm," IEEE Transactions on Antennas and Propagation, vol. 51, no. 9, pp. 2210-2217, 2003.

[3] B. P. Kumar and G. R. Branner, "Design of unequally spaced arrays for performance improvement," IEEE Transactions on Antennas and Propagation, vol. 47, no. 3, pp. 511-523, 1999.

[4] B. P. Kumar and G. R. Branner, "Generalized analytical technique for the synthesis of unequally spaced arrays with linear, planar, cylindrical or spherical geometry," IEEE Transactions on Antennas and Propagation, vol. 53, no. 2, pp. 621-634, 2005.

[5] K. Yang, Z. Zhao, and Y. Liu, "Synthesis of sparse planar arrays with matrix pencil method," in Proceedings of the International Conference on Computational Problem-Solving (ICCP '11), pp. 82-85, 2011.

[6] T. K. Sarkar and O. Pereira, "Using the matrix pencil method to estimate the parameters of a sum of complex exponentials," IEEE Antennas and Propagation Magazine, vol. 37, no. 1, pp. 4855, 1995.

[7] Y. Hua and T. K. Sarkar, "Matrix pencil method for estimating parameters of exponentially damped/undamped sinusoids in noise," IEEE Transactions on Acoustics, Speech, and Signal Processing, vol. 38, no. 5, pp. 814-824, 1990.

[8] Y. Hua, "Estimating two-dimensional frequencies by matrix enhancement and matrix pencil," IEEE Transactions on Signal Processing, vol. 40, no. 9, pp. 2267-2280, 1992.

[9] J. Wang and J. J. Zhou, "Modified MEMP method for 2D scattering center measurement based on GTD model," in Proceedings of the International Conference on Microwave and Millimeter Wave Technology (ICMMT '08), pp. 987-990, April 2008.

[10] C. Jian, S. Wang, and L. Lin, "2-D DOA estimation by MEMP based on L-shape array," in Proceedings of the 8th International Conference on Signal Processing (ICSP '06), vol. 1, pp. 16-20.

[11] Y. Liu, Z. Nie, and Q. H. Liu, "Reducing the number of elements in a linear antenna array by the matrix pencil method," IEEE
Transactions on Antennas and Propagation, vol. 56, no. 9, pp. 2955-2962, 2008.

[12] D. K. Cheng, "Optimization techniques for antenna arrays," Proceedings of the IEEE, vol. 59, no. 12, pp. 1664-1674, 1971.

[13] E. K. Miller and D. M. Goodman, "A pole-zero modeling approach to linear array synthesis I. The unconstrained solution," Radio Science, vol. 18, no. 1, pp. 57-69, 1983.

[14] S. Rouquette and M. Najim, "Estimation of frequencies and damping factors by two-dimensional ESPRIT type methods," IEEE Transactions on Signal Processing, vol. 49, no. 1, pp. 237245, 2001.

[15] Y. Yu and H. T. Hui, "Design of a mutual coupling compensation network for a small receiving monopole array," IEEE Transactions on Microwave theory and Techniques, vol. 59, no. 9, pp. 2241-2245, 2011.

[16] B. H. Wang, H. T. Hui, and M. S. Leong, "Decoupled 2D direction of arrival estimation using compact uniform circular arrays in the presence of elevation-dependent mutual coupling," IEEE Transactions on Antennas and Propagation, vol. 58, no. 3, pp. 747-755, 2010. 

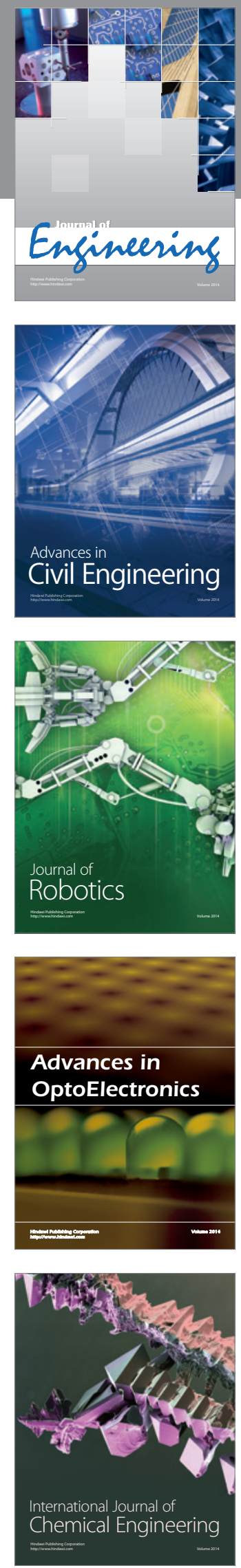

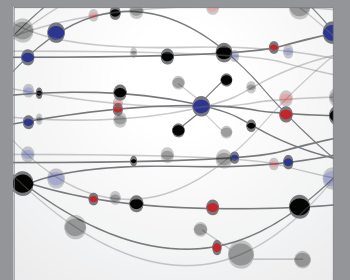

The Scientific World Journal
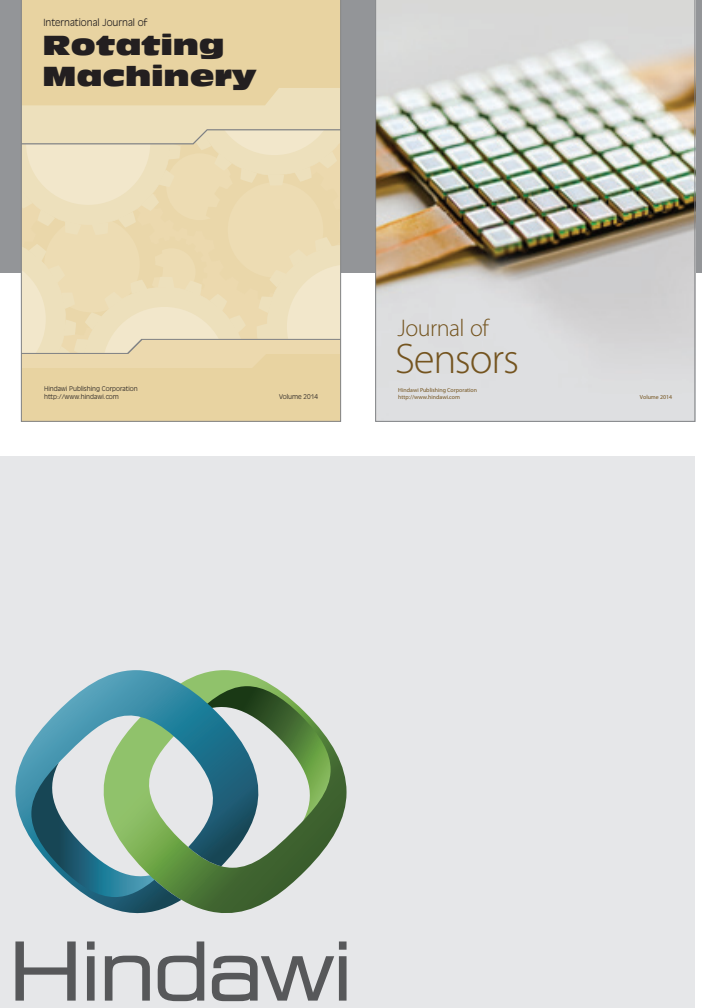

Submit your manuscripts at http://www.hindawi.com
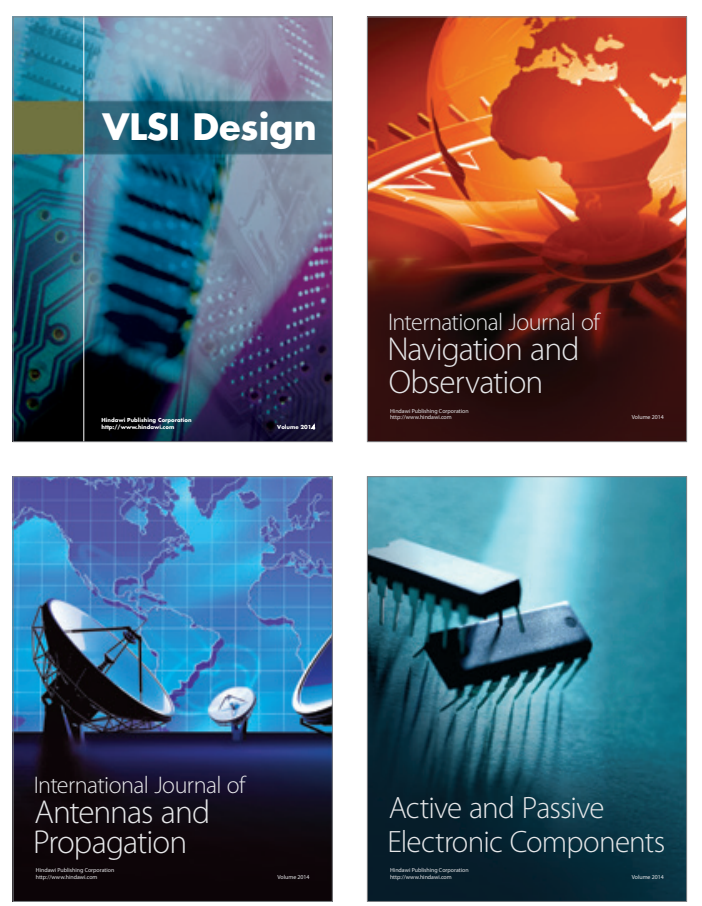
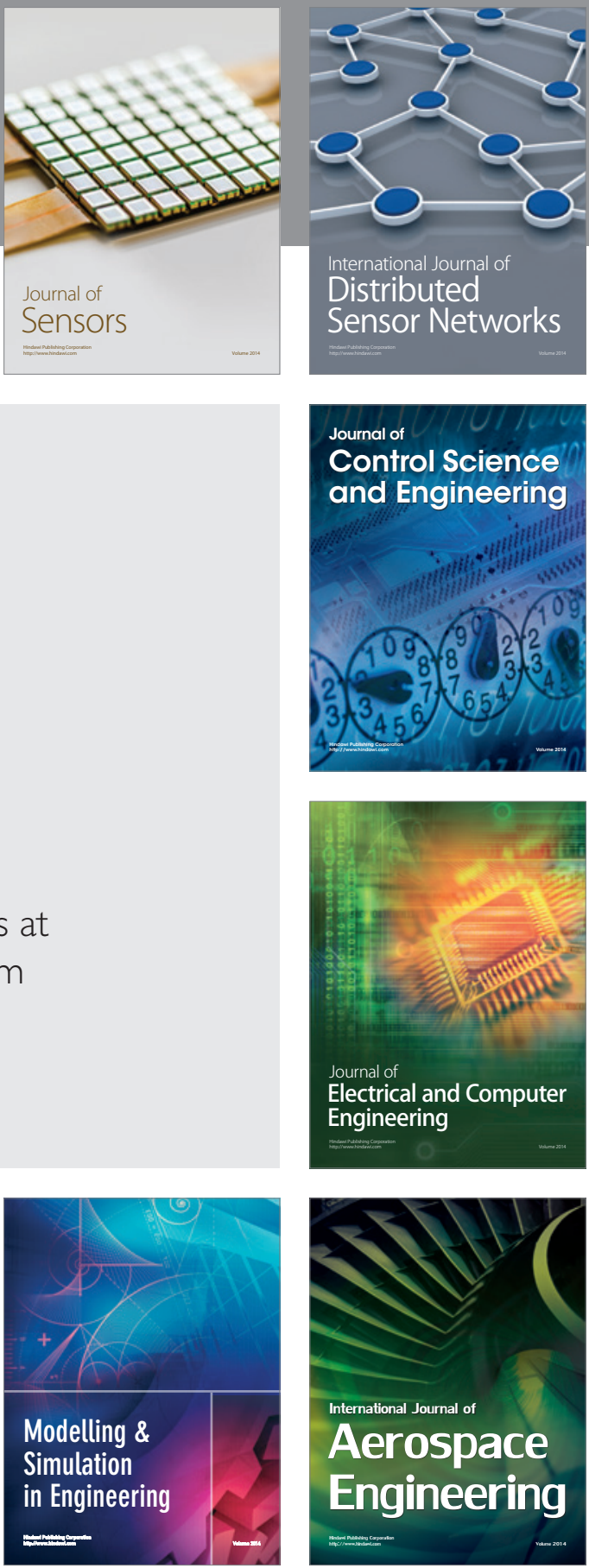

Journal of

Control Science

and Engineering
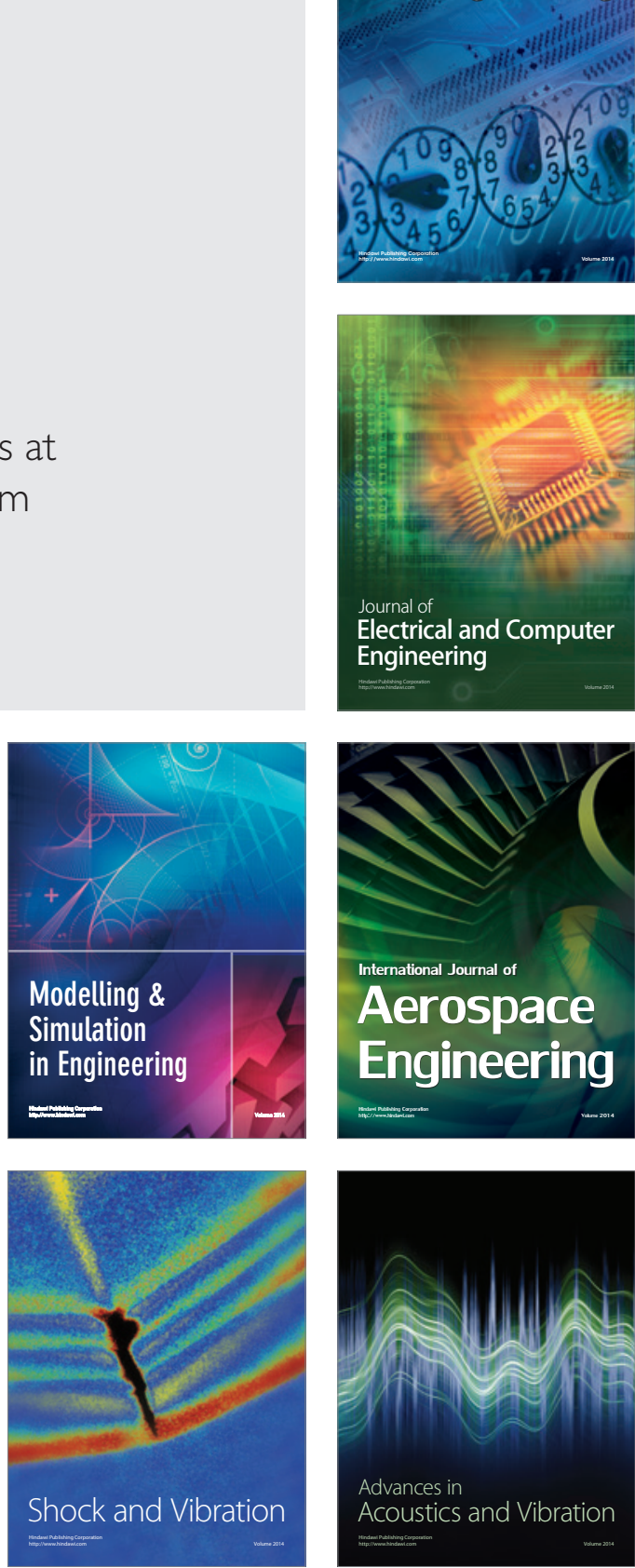